The University of Southern Mississippi

The Aquila Digital Community

Faculty Publications

9-7-2010

\title{
Biofunctionalization and Immobilization of a Membrane via Peptide Binding (CR3-1, S2) by a Monte Carlo Simulation
}

\author{
Ras B. Pandey \\ University of Southern Mississippi, ras.pandey@usm.edu \\ Hendrik Heinz \\ University of Akron, hendrik.heinz@uakron.edu \\ Jie Feng \\ University of Akron \\ Barry L. Farmer \\ Wright-Patterson Air Force Base, barry.farmer@wpafb.af.mil
}

Follow this and additional works at: https://aquila.usm.edu/fac_pubs

Part of the Astrophysics and Astronomy Commons

\section{Recommended Citation}

Pandey, R. B., Heinz, H., Feng, J., Farmer, B. L. (2010). Biofunctionalization and Immobilization of a Membrane via Peptide Binding (CR3-1, S2) by a Monte Carlo Simulation. Journal of Chemical Physics, 133(9).

Available at: https://aquila.usm.edu/fac_pubs/768

This Article is brought to you for free and open access by The Aquila Digital Community. It has been accepted for inclusion in Faculty Publications by an authorized administrator of The Aquila Digital Community. For more information, please contact Joshua.Cromwell@usm.edu. 


\section{Biofunctionalization and immobilization of a membrane via peptide binding $\left(\mathrm{CR}_{3}-1, \mathrm{~S} 2\right)$ by a Monte Carlo simulation}

Article in The Journal of Chemical Physics · September 2010

DOI: 10.1063/1.3484241 · Source: PubMed

CITATIONS

4

4 authors, including:

Ras B Pandey

University of Southern Mississippi

258 PUBLICATIONS 1,903 CITATIONS

SEE PROFILE

Barry L Farmer

Wright-Patterson Air Force Base

164 PUBLICATIONS 3,293 CITATIONS

SEE PROFILE
READS

37

Hendrik Heinz

University of Akron

60 PUBLICATIONS $\quad \mathbf{1 , 8 1 0}$ CITATIONS

SEE PROFILE

Some of the authors of this publication are also working on these related projects:

Project understanding high temperature metal corrosion in 4D View project 


\title{
Biofunctionalization and immobilization of a membrane via peptide binding (CR3-1, S2) by a Monte Carlo simulation
}

\author{
R. B. Pandey, ${ }^{1, a)}$ Hendrik Heinz, ${ }^{2}$ Jie Feng, ${ }^{2}$ and Barry L. Farmer ${ }^{3}$ \\ ${ }^{1}$ Department of Physics and Astronomy, University of Southern Mississippi, Hattiesburg, \\ Mississippi 39406, USA \\ ${ }^{2}$ Department of Polymer Engineering, University of Akron, Akron, Ohio 44325, USA \\ ${ }^{3}$ Air Force Research Laboratory, Materials and Manufacturing Directorate, AFRL/RXBP, \\ Wright-Patterson AFB, Ohio 45433, USA
}

(Received 22 April 2010; accepted 5 August 2010; published online 7 September 2010)

\begin{abstract}
A coarse-grained computer simulation model is used to study the immobilization of a dynamic tethered membrane (representation of a clay platelet) in a matrix of mobile peptide chains CR3-1: ${ }^{1}$ Trp $-{ }^{2}$ Pro $-{ }^{3}$ Ser $-{ }^{4}$ Ser $-{ }^{5} \mathrm{Tyr}-{ }^{6} \mathrm{Leu}-{ }^{7} \mathrm{Ser}-{ }^{8} \mathrm{Pro}-{ }^{9} \mathrm{Ile}-{ }^{10} \mathrm{Pro}-{ }^{11} \mathrm{Tyr}-{ }^{12} \mathrm{Ser} \quad$ and S2: ${ }^{1} \mathrm{His}-{ }^{2} \mathrm{Gly}-{ }^{3} \mathrm{Ile}-{ }^{4} \mathrm{Asn}-{ }^{5} \mathrm{Thr}-{ }^{6} \mathrm{Thr}-{ }^{7} \mathrm{Lys}-{ }^{8} \mathrm{Pro}-{ }^{9} \mathrm{Phe}-{ }^{10} \mathrm{Lys}-{ }^{11} \mathrm{Ser}-{ }^{12} \mathrm{Val}$ on a cubic lattice. Each residue interacts with the membrane nodes with appropriate interaction and executes their stochastic motion with the Metropolis algorithm. Density profiles, binding energy of each residue, mobility, and targeted structural profile are analyzed as a function of peptide concentration. We find that the binding of peptides S2 is anchored by lysine residues ( $\left.{ }^{7} \mathrm{Lys},{ }^{10} \mathrm{Lys}\right)$ while peptides CR3-1 do not bind to membrane. The membrane slows down as peptides (S2) continues to bind leading to its eventual pinning. How fast the immobilization of the membrane occurs depends on peptide concentration. Binding of peptide (S2) modulates the morphology of the membrane. The immobilization of membrane occurs faster if peptides (S2) are replaced by the homopolymer of lysine ([Lys $]_{12}$ of the same molecular weight), the strongest binding residue. The surface of membrane can be patterned with somewhat reduced roughness with the homopolymer of lysine than that with peptide (S2). ( $) 2010$ American Institute of Physics. [doi:10.1063/1.3484241]
\end{abstract}

\section{INTRODUCTION}

Immobilization of cells via adhesive peptides ${ }^{1}$ plays a very important role in cell adhesion to regenerate tissues such as corneal epithelium. ${ }^{2}$ Membrane and nanoparticles (e.g., gold and palladium) biofunctionalized by appropriate peptides have enormous potential in such applications as designing multifunctional materials for sensors, tissue engineering, regenerative medicine, and drug delivery to desired targets. $^{3-8}$ The effect of interaction of membrane surface with appropriate peptide in a desired tissue culture is critical in controlling the dynamics and morphology of the membrane and cell. A cell is too complex to consider its full functional characteristics in such a computer simulation study presented here. Therefore, we focus on the basic characteristics of a tethered membrane as it is immersed in a peptide solution.

The structural analysis of a tethered membrane including crumpling has been a subject of enormous interest for a long time. ${ }^{9-12}$ The dynamics of a tethered membrane has been extensively studied by computer simulation. ${ }^{13-15}$ A tethered membrane exhibits multiple dynamics ${ }^{13,14}$ over a range of time scales, e.g., short time $\left(R \propto t^{1 / 8}\right)$ to long time asymptotic diffusion $\left(R \propto t^{1 / 2}\right)$ where $R$ is the root mean square displacement of a local node and $t$ is the time step. What happens when the membrane is placed in a host matrix consisting of peptides? Obviously the concentration of peptide is very important in controlling the availability of free volume for the

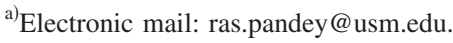

membrane to relax and execute its stochastic motion. In general, the higher the concentration of the underlying matrix, i.e., peptides, the longer the relaxation time is for constituents, i.e., peptides and membrane, to reach asymptotic dynamics. In addition to concentration, interaction between peptides and membrane may also affect the dynamics and self-assembly.

How does the membrane move and relax in presence of peptides that bind in comparison to those that do not? Such questions are relevant, for example, in the context to binding of peptides S2 $\left({ }^{1} \mathrm{His}-{ }^{2} \mathrm{Gly}-{ }^{3} \mathrm{Ile}-{ }^{4} \mathrm{Asn}-{ }^{5} \mathrm{Thr}-{ }^{6} \mathrm{Thr}\right.$ $-{ }^{7}$ Lys $-{ }^{8}$ Pro- $\left.-{ }^{9} \mathrm{Phe}-{ }^{10} \mathrm{Lys}-{ }^{11} \mathrm{Ser}-{ }^{12} \mathrm{Val}\right)$ and CR3-1 $\left({ }^{1}\right.$ Trp $-{ }^{2}$ Pro $-{ }^{3} \mathrm{Ser}-{ }^{4} \mathrm{Ser}-{ }^{5} \mathrm{Tyr}-{ }^{6} \mathrm{Leu}-{ }^{7} \mathrm{Ser}-{ }^{8} \mathrm{Pro}-$ ${ }^{9} \mathrm{Ile}-{ }^{10} \mathrm{Pro}-{ }^{11} \mathrm{Tyr}-{ }^{12} \mathrm{Ser}$ ) to clay platelets. ${ }^{6-8}$ We have examined the immobilization dynamics of the tethered membrane in the presence of peptides CR3-1 and S2 using a coarse-grained model, as described in the following.

\section{MODEL}

A cubic box of size $\mathrm{L}^{3}$ is considered as a host space. The membrane, corresponding to a coarse-grained description of a bendable clay platelet, ${ }^{13}$ is modeled by a set of nodes connected by flexible (fluctuating) bonds initially on a square grid. The membrane is placed at the center of the box followed by peptides inserted randomly in the box (see Fig. 1). The peptide chain ${ }^{16,17}$ is represented by a set of nodes, each representing the interaction characteristics of a corresponding amino acid, which are tethered by fluctuating covalent 


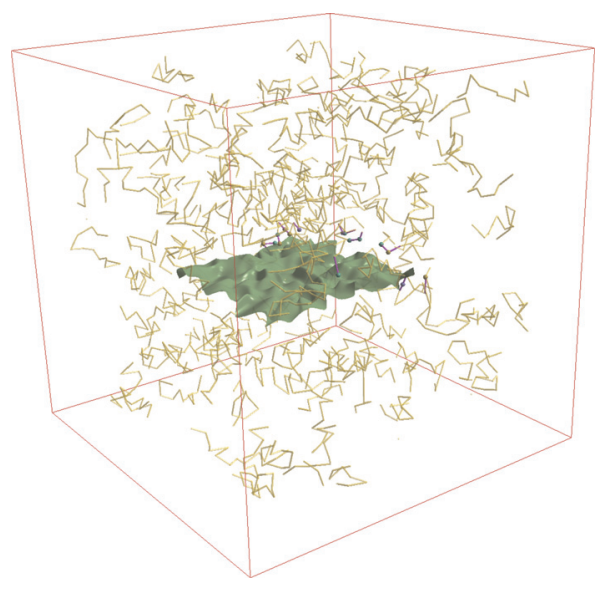

FIG. 1. A tethered membrane at the center surrounded by peptide chains on a cubic lattice.

bonds. A node in both membrane and peptide occupies a unit cube i.e., its eight lattice sites; the bond length between consecutive nodes can vary (fluctuate) between 2 and $\sqrt{ } 10$ with an exception of $\sqrt{ } 8$ in unit of lattice constant. Such a bondfluctuation description is known to be computationally efficient while incorporating ample degrees of freedom in complex polymer systems ${ }^{18}$ and multicomponent nanocomposites. $^{19,20}$

We consider a set of interactions among residues of the peptide (intrachain and interchain) and with the membrane nodes within a range of interaction. Only the excluded volume interaction is however considered between nodes pertaining to the membrane. Since each peptide node represents a specific residue, it is essential to capture their specificity or uniqueness. The specificity of each residue is captured by an interaction matrix (see below) method recently used to study adsorption of peptides on gold and palladium substrates. ${ }^{16}$ This approach is briefly described as follows. Twenty amino acids can be divided into three broad categories: hydrophobic $(H)$, hydrophilic or polar $(P)$, and electrostatic $(E)$. An amino acid within each group is further distinguished by its relative hydrophobicity, polar, or electrostatic strength. In addition to peptide chains, there is a tethered membrane $(S)$. This constitutes four main components, $H, P, E$, and $S$ in which there are three subgroups to characterize specificity of each residue: ${ }^{16}$ eight hydrophobic $\left(H_{1}, H_{2} \cdots H_{8}\right)$, eight polar $\left(P_{1}, P_{2} \cdots P_{8}\right)$, and four electrostatic $\left(E_{1}, E_{2}, E_{3}, E_{4}\right)$. Each node (membrane and peptide) executes stochastic movement with the Metropolis algorithm using a set of phenomenological interactions among these components as follows.

Interaction matrix. Interaction among four main interacting components $(H, P, E, S)$ can be represented by a $4 \times 4$ matrix. ${ }^{16}$ The interaction strength between two elements (nodes) at sites $i$ and $j$ separated by a distance $r_{i j}$ is represented by the standard Lennard-Jones potential

$$
U_{i j}=f \varepsilon_{i j}\left[\left(\frac{\sigma}{r_{i j}}\right)^{12}-\left(\frac{\sigma}{r_{i j}}\right)^{6}\right], \quad r_{i j}<r_{c},
$$

where $r_{c}=\sqrt{ } 8$ (in unit of lattice constant) is the cutoff range and $f \varepsilon_{i j}$ is the interaction strength with an arbitrary parameter $f$ that can be varied and $\sigma=1$. On a cubic lattice the
TABLE I. A typical interaction matrix among $H, P, E$, and $S$ components.

\begin{tabular}{lcccr}
\hline \hline & $H$ & $P$ & $E$ & \multicolumn{1}{c}{$S$} \\
\hline$H$ & 0.0 & 0.0 & 0.0 & 0.1 \\
$P$ & 0.0 & -0.2 & -0.2 & -0.2 \\
$E$ & 0.0 & -0.2 & $\varepsilon_{E E}$ & -0.5 \\
$S$ & 0.1 & -0.2 & -0.5 & 0.0 \\
\hline \hline
\end{tabular}

distance between two sites is discrete and measured in units of the lattice constant. Note that the minimum distance between two neighboring particles is two in units of the lattice constant. The depth of the LJ potential is controlled by the magnitude of the pair interaction strength $f \varepsilon_{i j}$. ${ }^{16}$ The number of the pair interaction matrix elements is however reduced somewhat due to symmetries, e.g., $\varepsilon_{i j}=\varepsilon_{j i}$.

The magnitude of each interaction elements is based on the insight gained from all atomistic description and their known general characteristics. ${ }^{8,16}$ A typical set of values of the interaction matrix is presented in Table I. The interaction matrix elements ${ }^{16}$ for residues are obtained by weighing the broad interaction strength, $\varepsilon_{H S}, \varepsilon_{P S}$, and $\varepsilon_{E S}$, appropriately by their specific interaction characteristics, i.e., by their relative hydrophobicity and polar and electrostatic strengths within each group. This leads to a larger interaction matrix with $\varepsilon_{H 1 S}, \varepsilon_{H 2 S}, \cdots \varepsilon_{H 8 S}, \varepsilon_{P 1 S}, \varepsilon_{P 2 S}, \cdots \varepsilon_{P 8 S}, \varepsilon_{E 1 S}, \varepsilon_{E 2 S}, \cdots$ $\varepsilon_{E 4 S}$. We have used $f=100$ the interaction energy factor to accentuate the differences in adsorption (binding) probabilities for CR3-1 and S2 peptides. Interactions ${ }^{16}$ among the four electrostatic residues $\left(\varepsilon_{E E}\right.$ in Table I) are $\varepsilon_{11}=\varepsilon_{12}=\varepsilon_{22}=\varepsilon_{33}$ $=\varepsilon_{34}=\varepsilon_{44}=0.1$ and $\varepsilon_{13}=\varepsilon_{14}=\varepsilon_{23}=\varepsilon_{24}=-0.4$.

Incorporating the specificity of individual amino acid group for a particular system enhances the size of the interaction matrix. ${ }^{16}$ The choices of the matrix elements presented here are primarily to illustrate how to predict the relative binding of peptides CR3-1 and S2 with the clay platelet using the coarse-grained model with phenomenological interactions. The relative hydrophobic interaction strength of S2 $\left({ }^{1} \mathrm{His}-{ }^{2} \mathrm{Gly}-{ }^{3} \mathrm{Ile}-{ }^{4} \mathrm{Asn}-{ }^{5} \mathrm{Thr}-{ }^{6} \mathrm{Thr}-{ }^{7} \mathrm{Lys}-\right.$ ${ }^{8}$ Pro- $\left.-{ }^{9} \mathrm{Phe}-{ }^{10} \mathrm{Lys}-{ }^{11} \mathrm{Ser}-{ }^{12} \mathrm{Val}\right)$ can be represented by $\quad\left({ }^{1} P_{6}-{ }^{2} H_{8}-{ }^{3} H_{1}-{ }^{4} P_{8}-{ }^{5} P_{1}-{ }^{6} P_{1}-{ }^{7} E_{3}-{ }^{8} P_{5}-\right.$ $\left.{ }^{9} \mathrm{H}_{4}-{ }^{10} \mathrm{E}_{3}-{ }^{11} \mathrm{P}_{2}-{ }^{12} \mathrm{H}_{2}\right)$ and that of CR3-1 ${ }^{1} \mathrm{Trp}-$ ${ }^{2} \mathrm{Pro}-{ }^{3} \mathrm{Ser}-{ }^{4} \mathrm{Ser}-{ }^{5} \mathrm{Tyr}-{ }^{6} \mathrm{Leu}-{ }^{7} \mathrm{Ser}-{ }^{8} \mathrm{Pro}-{ }^{9} \mathrm{Ile}-{ }^{10} \mathrm{Pro}$ $\left.-{ }^{11} \mathrm{Tyr}-{ }^{12} \mathrm{Ser}\right)$ by $\left({ }^{1} P_{3}-{ }^{2} P_{5}-{ }^{3} P_{2}-{ }^{4} P_{2}-{ }^{5} P_{4}-{ }^{6} H_{3}-\right.$ $\left.{ }^{7} P_{2}-{ }^{8} P_{5}-{ }^{9} H_{1}-{ }^{10} P_{5}-{ }^{11} P_{4}-{ }^{12} P_{2}\right)$.

Stochastic movement. Each residue and membrane node executes their stochastic movement ${ }^{13,19,20}$ with the Metropolis algorithm. A particle (node) at a site $i$ and one of its 26 neighboring sites $j$ are selected randomly. If site $j$ is empty and the change in the bond length with the proposed move is within the allowed range, then an attempt is made to move it from site $i$ to site $j$. Provided the excluded volume condition is satisfied, the energy in old $\left(E_{i}\right)$ and new configuration $\left(E_{j}\right)$ is compared and particle is moved from site $i$ to $j$ with probability $\exp \left(-\Delta E_{i j} / T\right)$, where $\Delta E_{i j}=E_{j}-E_{i}$, the temperature $T$ is in unit of the Boltzmann constant and the interaction energy and fixed to unity here. Attempts to move each node once defines one Monte Carlo step time. Simulation is performed for a sufficiently long time to achieve equilibration. 

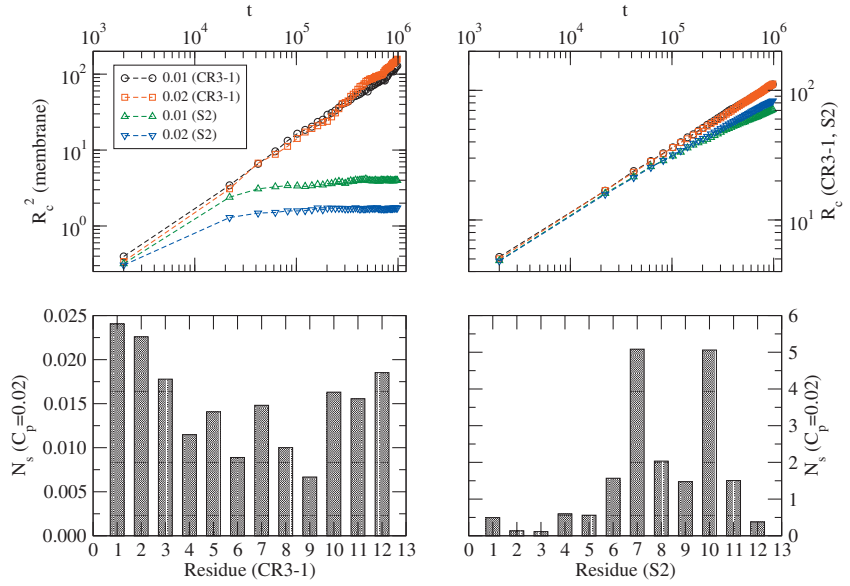

FIG. 2. Top row: variation of the rms displacement $\left(R_{c}\right)$ of the center of mass of the membrane (left) and that of the peptides (right) with the time steps for peptide concentrations 0.01 and 0.02 . Bottom row: average number of membrane sites around each residue in CR3-1 (left) and S2 (right) with the peptide concentration 0.02 . Membrane of size $16^{2}$ is used on a $64^{3}$ lattice with ten independent samples.

A number of physical quantities are evaluated during the simulation including energy of each residue, their mobility, average number of components (membrane nodes and residues) within the range of interaction, and the variation of the mean square displacement of the center of mass of the peptide chains and that of the membrane with the time steps which are presented in the following.

\section{RESULTS AND DISCUSSION}

Starting with a homogeneous solution of the peptides containing the membrane, the immobilization process was monitored in the simulation. In the following, we report on the displacement of the membrane and of the peptides, their binding strength, and mobility upon biofunctionalization. The method also allowed the variation of peptide concentration to broaden the analysis.

Let us first consider the variation of the root mean square displacement $\left(R_{c}\right)$ of the center of mass of the membrane and that of the CR3-1 and S2 peptides as shown in Fig. 2 (top row). The slope of $R_{c}$ versus time step $(t)$ is the measure of the speed by which membrane and peptide move. We see that the membrane moves much faster in presence of CR3-1 than in the presence of S2. As the time progresses, the membrane becomes immobilized in the presence of S2 peptides due to binding. The membrane continues diffusion across the time range in the presence of CR3-1 which binds weaker and has hardly any effect. The average mobility of the peptides is much less affected than that of the membrane due to a majority of free S2 peptides even after tethering of many to the membrane. However, some reduction in average mobility in the long time regime is clearly reflected due to the tether of many S2 molecules to the membrane.

In order to quantify the relative binding of peptides, we evaluate the average number $\left(\mathrm{N}_{\mathrm{s}}\right)$ of membrane sites within the range of interaction to each residue of both CR3-1 and S2 in Fig. 2 (bottom row). The number of binding sites of the membrane to $\mathrm{S} 2$ is overwhelmingly larger than CR3-1 which suggests a much higher probability of binding of S2 than binding of CR3-1. Note that the binding of S2 to the membrane is mediated by lysine residues $\left({ }^{7} \mathrm{Lys},{ }^{10} \mathrm{Lys}\right)$ which retain the largest number of membrane sites within their interaction range over time. As we have identified that $\mathrm{S} 2$ binds to the membrane (consistent with recent experiments), ${ }^{7}$ we will focus on the effect of its binding on the immobilization of the membrane in the following.

In Fig. 3, we present the evolution of peptide assembly through representative snapshots along with associated density profiles normal to the initial membrane plane for the membrane and the S2 peptide. Increasing self-organization can be seen as a function of time steps and the number of peptide chains that can bind to the membrane increases with the number of MC steps. The increase in the density of peptides around the membrane with time is also illustrated by the density profile. It should be pointed out that each component (membrane and peptides) executes their stochastic motion; the dynamics of membrane changes as peptides bind as reflected in the variation of the root mean square (rms) displacement of the center of mass of membrane (see Fig. 2, top row).

Let us examine closely how the peptide binding and its concentration affect the dynamics of the membrane. As seen above (Fig. 2, top row), the global dynamics of the membrane can be studied by analyzing the variation of the rms displacement $\left(R_{c}\right)$ of its center of mass with the number of time steps. Figure 4 shows more details of this behavior for a range of higher concentrations of the $\mathrm{S} 2$ peptide. Initially, $R_{c}$ increases with the time step with a well-defined power-law, i.e., $R_{c} \propto t^{\nu}$, where the exponent $\nu$ characterizes the dynamics. The onset of slower motion of the center of mass of the membrane is clearly seen as time progresses and as the number of binding peptides increases. The motion of the membrane pinned down to a nearly stand-still with the binding of a sufficiently large number of peptides at later time steps. The dynamics of the membrane depends on the concentration of the peptide: at higher peptide concentration, (i) the characteristic initial dynamics is slower with lower $\nu$ and (ii) pinning (immobilization) of the membrane occurs faster. How fast immobilization of membrane occurs and how much its local (segmental) dynamics slows down is thus dictated by the amount of peptide (S2) that can effectively bind. Therefore, both immobilization and segmental mobility of membrane can be controlled by the concentration of appropriate peptides.

At lower concentration, most of the $\mathrm{S} 2$ peptides can bind to the membrane in a sufficient span of time. At high peptide concentration, there may still be a majority of free peptides in the sample. As a result, the average mobility of residues in peptides should be higher at higher peptide concentrations. Figure 5 shows the mobility profiles of residues in S2 at higher concentrations $(0.10,0.20)$ at different time steps $\left(10^{3}, 10^{4}\right.$, and $\left.10^{5}\right)$. Mobility of a residue is defined as the average number of moves per unit time steps. We understand that the average residue mobility decreases with time steps due to the tethering of many peptides to the surface (Fig. 5). However, the mobility of the residue at the ends of the peptide chains is nearly constant and less affected by binding. As the most strongly binding residues ${ }^{7}$ Lys and ${ }^{10}$ Lys are lo- 

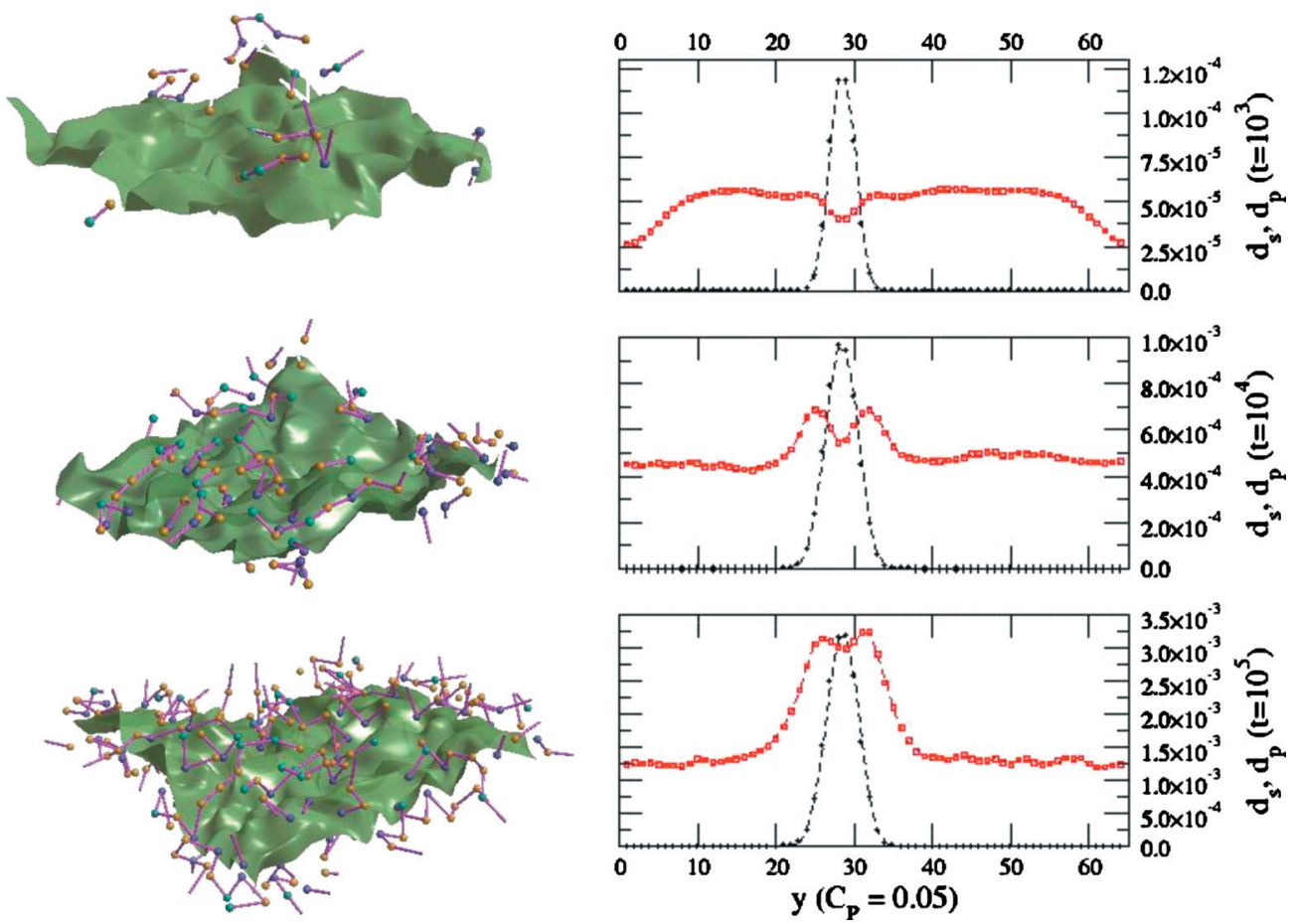

FIG. 3. Left: snapshots of the membrane and parts of bound S2 peptide chains within the range of interaction of the membrane for increasing number of time steps $t=10^{3}, 10^{4}$, and $10^{5}$ (from top to bottom). Right: corresponding vertical density profile of the membrane (circles) and peptides (squares), showing the accumulation of peptide near the membrane as time proceeds. A membrane of size $16^{2}$ was used on a $64^{3}$ lattice with a peptide concentration of 0.05 and 100-1000 independent samples.

cated in the interior of the chain (also ${ }^{4}$ Ser binds), the mobility of terminal residues is likely less affected by binding. Also, constraints due to covalent bonds, intrachain, and interchain interactions between amino acids residues tend to reduce the mobility of interior residues more than that of the end groups at such higher peptide concentrations. At such a high concentrations, there are ample numbers of free peptides with many end groups.

We have now shown that peptide-functionalization and dynamics of a membrane depend on the type and concentration of the peptides. Since S2 peptides are primarily anchored to the membrane through ${ }^{7}$ Lys and ${ }^{10}$ Lys residues, it

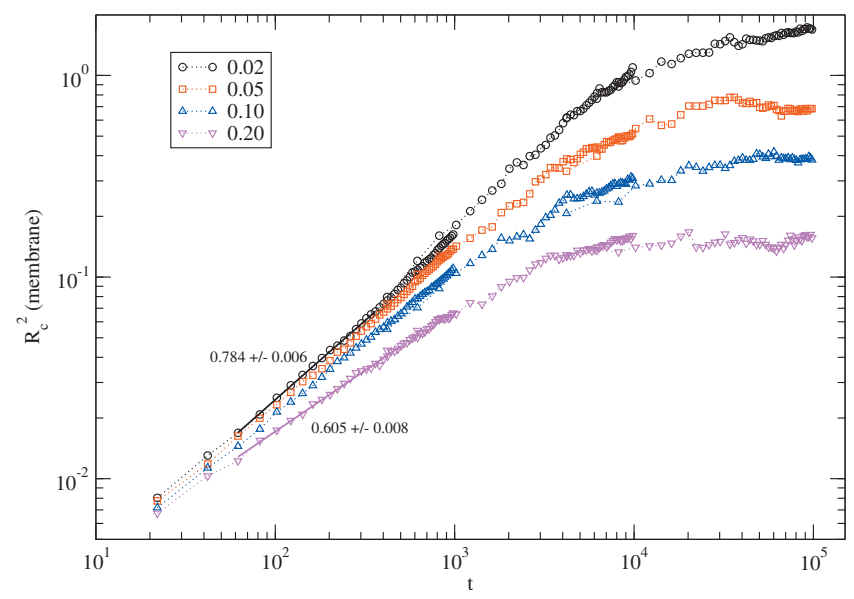

FIG. 4. Mean square displacement of the center of mass of the membrane $\left(16^{2}\right)$ vs number of MC steps in a solution of peptide (S2) with concentrations $0.02,0.05,0.10$, and 0.20 on a $64^{3}$ lattice. Slopes of the initial data sets at the lowest $(0.02)$ and highest $(0.20)$ concentrations are indicated. Twenty to one hundred independent samples were used. would be interesting to explore what happens for a homopeptide of lysine as an extreme limit for attractive interactions. Figure 6 shows snapshots at the end of simulations for different concentrations of peptide S2 and peptide $[\mathrm{Lys}]_{12}$ in the range 0.02-0.20. Apparently, the amount of bound materials increases with higher concentration. However, the ways S2 and polylysine peptides bind (adsorb) to the membrane differ substantially. Since each of the 12 lysine residues in Lys 12 is strongly attracted to the membrane in contrast with only two residues $\left({ }^{7}\right.$ Lys, ${ }^{10}$ Lys) in peptide $\mathrm{S} 2$, the $\mathrm{Lys}_{12}$ chains are adsorbed linearly along the bulk of the membrane surface. Polylysine on the membrane surface thus exhibits a rather orderly pattern in contrast with that of the $\mathrm{S} 2$ peptides which bind with fewer residues per chain and a higher number of chains. The adsorption of polylysine appears to influence the morphology of the membrane surface more than the adsorption of S2 peptides (see Fig. 6), i.e., the local movement of the membrane is constrained more by polylysine than by $\mathrm{S} 2$. The surface of the immobilized membrane tends to be smoother in the presence of tethered $\mathrm{Lys}_{12}$ and rougher in the presence of tethered S2 peptide, much like a specific foot print of each peptide. Differences in surface morphology are clearly associated with different adsorption characteristics of the two peptides, and the surface morphology of the membrane can thus be controlled by appropriately positioning anchoring residues in a specific sequence.

Based on visual inspection of the snapshots in Fig. 6, the global dynamics of the membrane is also expected to differ in the presence of polylysine in comparison to that in the presence of S2 peptide. Figure 7 shows the variation of the rms displacement of the membrane in the presence of peptide (S2) and polymer $\left([\mathrm{Lys}]_{12}\right)$ as a function of MC steps. For 

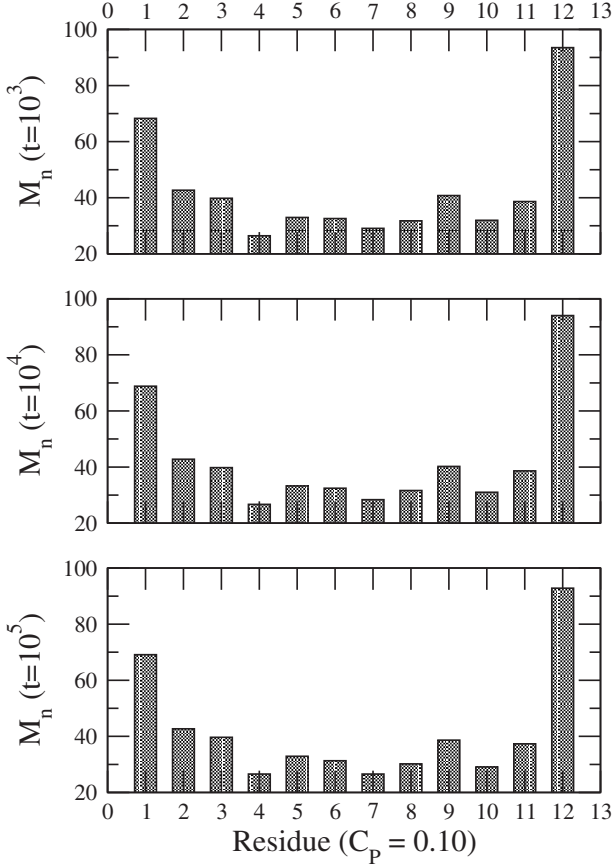
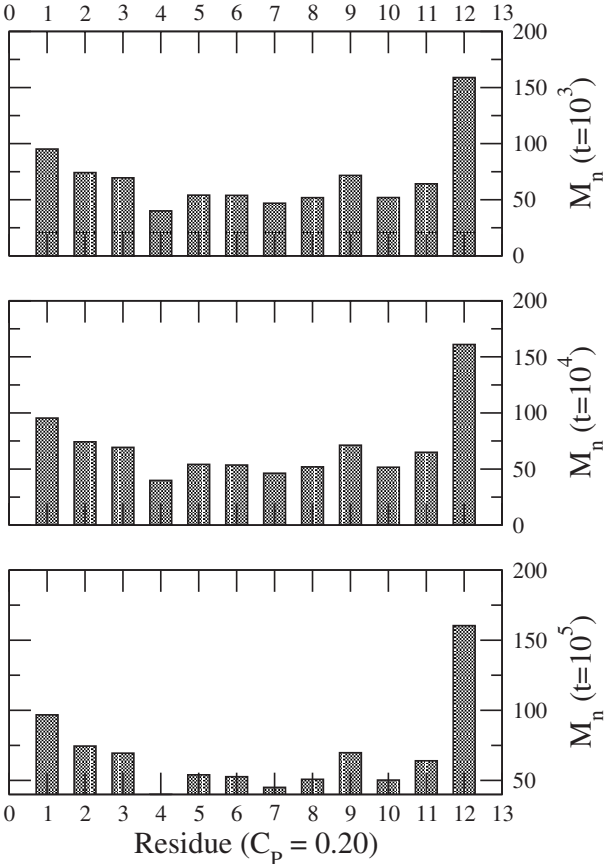

FIG. 5. Average mobility of residues in S2 with the peptide concentrations 0.10 and 0.20 at time steps $t=10^{3}, 10^{4}$, and $10^{5}$. Statistics is the same as in Fig. 4.

any equal concentration, the membrane becomes immobilized faster in the presence of polylysine. Thus, one can control the mobility and immobilization of membrane and its surface morphology by peptides with appropriate residues.

\section{CONCLUSIONS}

Monte Carlo simulations were performed to study the dynamics of a flexible membrane in the presence of the peptides CR3-1, S2, and polylysine. A specific coarse-grain model was employed for the peptides and for the membrane, which corresponds to a bendable clay platelet. Peptides and membrane execute their stochastic motion driven by thermodynamics under variation of the peptide concentration. The CR3-1 peptides do not significantly bind to the membrane which continues to perform its stochastic motion and diffuse along with the peptides with least resistance. The S2 peptides

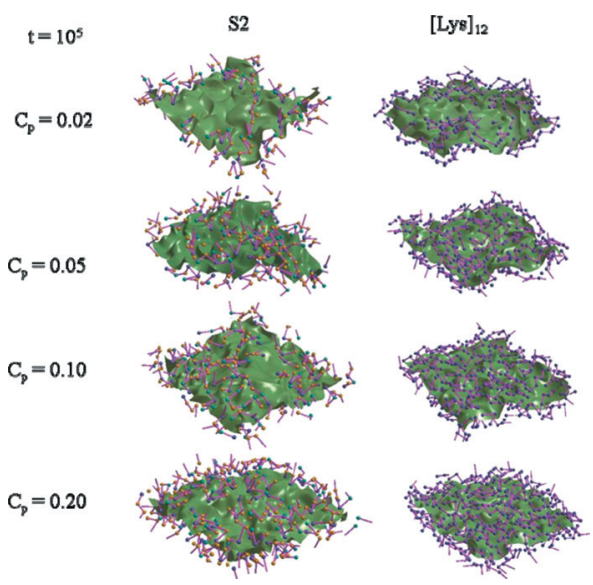

FIG. 6. Snapshots of membrane and S2 peptide (left) and membrane and Lys $_{12}$ peptide (right) at time step $10^{5}$ as a function of increasing peptide concentration. bind readily to membrane sites anchored by its lysine residues ${ }^{7}$ Lys and ${ }^{10}$ Lys which is consistent with recent experiments. The global motion of the membrane slows down considerably when $\mathrm{S} 2$ peptides continue to bind leading to its immobilization with increasing number of time steps. The duration to pin down the membrane by $\mathrm{S} 2$ peptides decreases with increasing concentration of the peptide. This seems consistent with the observation of coimmobilization of cells by cell adhesion peptides by Hatakeyama et al. ${ }^{1}$ Thereby, nonbinding peptides such as CR3-1 do not significantly affect conformation and dynamics in contrast with binding peptides such as $\mathrm{S} 2$.

The sequence of binding residues in the peptides not only modulates the immobilization dynamics but also the surface structure of the immobilized membrane. In our example, Lys residues are strongly attracted to the membrane

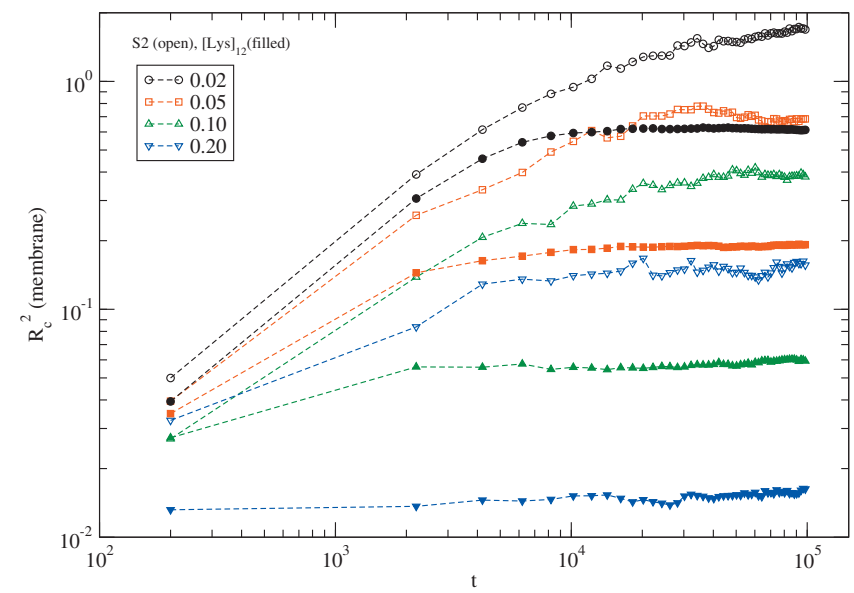

FIG. 7. Mean square displacement of the center of mass of the membrane vs MC time steps in the presence of S2 and $\mathrm{Lys}_{12}$ for a range of peptide concentrations from 0.02 to 0.20 . Statistics is the same as in Fig. 4 . 
(clay) surface, so that polylysine $\mathrm{Lys}_{12}$ immobilizes the membrane faster and patterns the membrane surface with somewhat lower roughness compared to the S2 peptide which only contains two Lys residues in the backbone. Therefore, the immobilization dynamics of a membrane, its surface morphology, and the formation of specific biomolecular patterns on the surface can be controlled by designing peptide sequences with appropriately distributed binding residues.

\section{ACKNOWLEDGMENTS}

Support from the Materials and Manufacturing Directorate of the Air Force Research Laboratory is gratefully acknowledged.

${ }^{1}$ H. Hatakeyama, A. Kikuchi, M. Yamato, and T. Okano, Biomaterials 27, 5069 (2006)

${ }^{2}$ K. Nishida, M. Yamato, Y. Hayashida, K. Watanabe, K. Yamamoto, E. Adachi, S. Nagai, A. Kikuchi, N. Maeda, H. Watanabe, T. Okano, and Y. Tano, N. Engl. J. Med. 351, 1187 (2004).

${ }^{3}$ M. Ebara, M. Yamato, T. Aoyagi, A. Kikuchi, K. Sakai, and T. Okano, Biomacromolecules 5, 505 (2004).

${ }^{4}$ M. Ebara, M. Yamato, T. Aoyagi, A. Kikuchi, K. Sakai, and T. Okano,
Tissue Eng. 10, 1125 (2004)

${ }^{5}$ C. Tamerler and M. Sarikaya, ACS Nano 3, 1606 (2009).

${ }^{6}$ M. B. Dickerson, K. H. Sandhage, and R. R. Naik, Chem. Rev. (Washington, D.C.) 108, 4935 (2008).

${ }^{7}$ L. F. Drummy, S. E. Jones, R. B. Pandey, B. L. Farmer, R. A. Vaia, and R. R. Naik, ACS Appl. Mater. Interfaces 2, 1492 (2010).

${ }^{8}$ H. Heinz, H. Koerner, R. A. Vaia, K. L. Anderson, and B. L. Farmer, Chem. Mater. 17, 5658 (2005).

${ }^{9}$ Y. Kantor, M. Kardar, and D. R. Nelson, Phys. Rev. Lett. 57, 791 (1986).

${ }^{10}$ F. F. Abraham, W. E. Rudge, and M. Plischke, Phys. Rev. Lett. 62, 1757 (1989).

${ }^{11}$ Statistical Mechanics of Membranes and Interfaces, edited by D. R. Nelson, T. Piran, and S. Weinberg (World Scientific, Singapore, 1989).

${ }^{12}$ J.-S. Ho and A. Baumgartner, Phys. Rev. Lett. 63, 1324 (1989).

${ }^{13}$ R. B. Pandey, K. L. Anderson, and B. L. Farmer, Phys. Rev. E 75, 061913 (2007).

${ }^{14}$ H. Popova and A. Milchev, Phys. Rev. E 77, 041906 (2008).

${ }^{15}$ S. T. Knauert, J. F. Douglas, and F. W. Starr, Macromolecules 43, 3438 (2010).

${ }^{16}$ R. B. Pandey, H. Heinz, J. Feng, B. L. Farmer, J. M. Slocik, L. F. Drummy, and R. R. Naik, Phys. Chem. Chem. Phys. 11, 1989 (2009).

${ }^{17}$ R. B. Pandey and B. L. Farmer, J. Chem. Phys. 130, 044906 (2009).

${ }^{18}$ I. Carmesin and K. Kremer, Macromolecules 21, 2819 (1988).

${ }^{19}$ R. B. Pandey and B. L. Farmer, Macromol. Theory Simul. 17, 208 (2008).

${ }^{20}$ R. B. Pandey and B. L. Farmer, J. Polym. Sci., Part B: Polym. Phys. 46, 2696 (2008) 